\title{
Synthesis of Ni base nanoparticles by W/O emulsion combustion
}

\author{
Jun Kobayashi $^{1} \mathbb{D} \cdot$ Noriyuki Kobayashi $^{2} \cdot$ Yoshinori Itaya $^{3} \cdot$ Masanobu Hasatani $^{4}$
}

Received: 14 April 2019 / Accepted: 20 June 2019 / Published online: 4 July 2019

(c) The Author(s) 2019

\begin{abstract}
To develop a process of fine particle production by spray pyrolysis, spray combustion of W/O (water-in-oil) emulsion, of which water phase was raw material solution and oil phase was fuel for heat source of high-temperature reaction field, was investigated. In this study, nickel oxide particles, which were a preliminary step of the nickel fine particle production, were synthesized and its structural characteristics were evaluated. Mixed solution of nickel nitrate and white kerosene was used as raw material. W/O emulsions were prepared using ultrasonic homogenizer and stirring these raw materials adding a surfactant. These emulsions were burning in a high temperature furnace to produce nickel oxide particles. The mean particle diameter of produced particles was less than $20 \mathrm{~nm}$ according to TEM observation. The diameter of the particles was much smaller than the estimated value based on the size distribution of dispersed solution phase in the emulsions and its concentration. Moreover, there is no effect of the concentration of the aqueous solution phase. On the other hand, X-ray diffraction pattern showed that the produced particles were complex of metal nickel with nickel oxide.
\end{abstract}

\section{Graphic abstract}

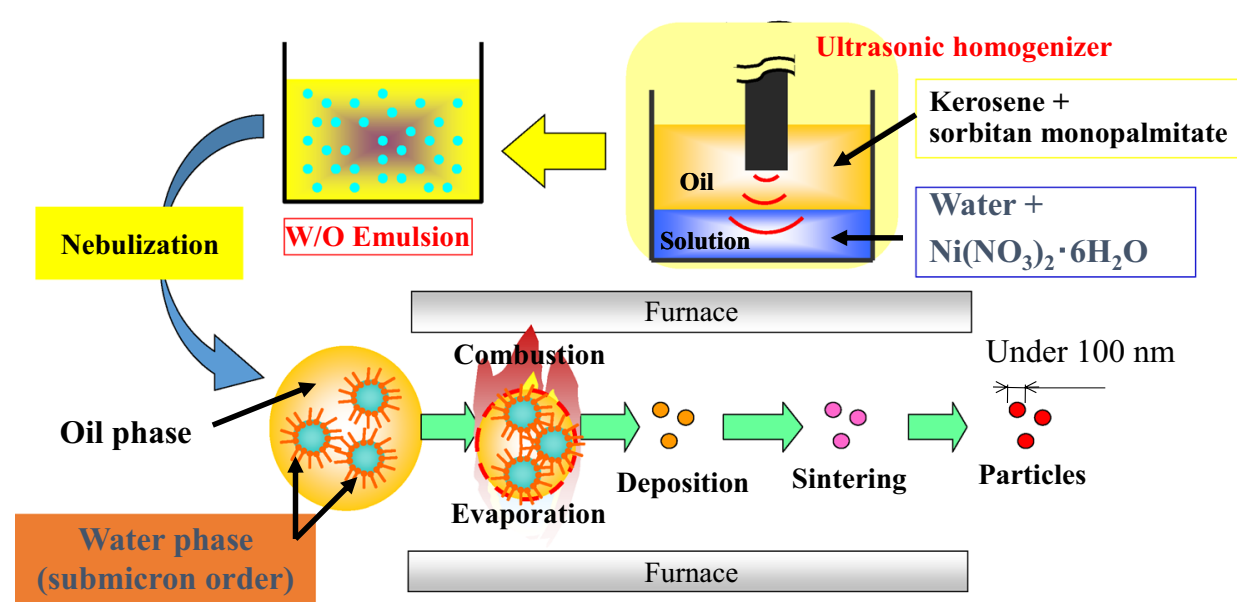

Keywords Nickel oxide nanoparticle $\cdot \mathrm{W} / \mathrm{O}$ emulsion $\cdot$ Spray combustion $\cdot$ Ultrasonic homogenizer

Jun Kobayashi

jkoba@cc.kogakuin.ac.jp

1 Department Mechanical Engineering, Kogakuin University, 1-24-2 Nishi-shinjuku, Shinjuku-ku, Tokyo 163-8677, Japan

2 Department Material Science, Nagoya University, Furo-cho, Chikusa-ku, Nagoya 464-8603, Japan

3 Department Mechanical Engineering, Gifu University, 1-1 Yanagido, Gifu 501-1193, Japan

4 Aichi Institute of Technology, 1247 Yachigusa, Yakusa-cho, Toyota 470-0392, Japan

\author{
List of symbols \\ $D_{\text {em }} \quad$ Diameter of water phase in W/O emulsion (m) \\ $D_{\mathrm{p}} \quad$ Diameter of produced particle (m) \\ $F_{\text {em1 }}$ Flow rate of emulsion injected into an atomization \\ nozzle $\left(\mathrm{m}^{3} \mathrm{~s}^{-1}\right)$ \\ $F_{\text {em2 }} \quad$ Flow rate of emulsion returned from an atomization \\ nozzle $\left(\mathrm{m}^{3} \mathrm{~s}^{-1}\right)$ \\ $M_{\mathrm{p}} \quad$ Molecular weight of produced particle $\left(\mathrm{kg} \mathrm{kmol}^{-1}\right)$ \\ $w \quad$ Weight of sample $(\mathrm{kg})$
}




$\begin{array}{ll}w_{0} & \text { Initial weight of sample }(\mathrm{kg}) \\ x & \text { Concentration of nickel salt solution }\left(\mathrm{kmol} \mathrm{m}^{-3}\right) \\ \rho_{\mathrm{p}} & \text { Density of produced particle }\left(\mathrm{kg} \mathrm{m}^{-3}\right)\end{array}$

\section{Introduction}

Spray pyrolysis is one of the methods for fine particle production [1-3] and a droplet of precursor solution or slurry is a template of a produced particle. Many studies for development of fine particle production using the method have already been reported. A spherical droplet of precursor solution or slurry almost decides the size of the produced particle when these droplets do not break and/or unite themselves. Therefore, spray methods to produce fine and uniform droplets have been studied on and mass production methods of these droplets have been developed. [4] Electrostatic spray [5-7], ultrasonic spray $[8,9]$ and low-pressure spray methods $[10,11]$ are suitable for uniform nano-particle production since fine and uniformly sized droplets are obtained. On the other hand, it has been difficult to generate a large amount of fine droplets using these spray methods. Therefore, there is a need to produce a large amount of nano-particles more effectively and industrially. Then, we proposed production method for the ultrafine particle by the combustion of W/O emulsion prepared by ultrasonic homogenizer [12]. In this method, a large amount of W/O emulsion that included fine and uniform droplets of a metallic salt solution can be fed to a reactor at a time. The emulsion is burned and high-temperature combustion field is formed in the reactor. As a result, fine water phases of the aqueous solution are dried, decomposed and sintered by thermal energy of combustion in a short time. Although particle production by W/O emulsion combustion has already been reported [13-15], the W/O emulsions have been prepared by simple stirring and the size of water phases is not small enough for nano-particle production of less than $1 \mathrm{~m} \mathrm{[16].} \mathrm{Furthermore,} \mathrm{there} \mathrm{has} \mathrm{been}$ little reporting of the relationship between particle morphology and water phase structure in the emulsion.

In this work, nano-particle production due to spray combustion of a W/O emulsion prepared with an ultrasonic homogenizer was performed to obtain the nickel oxide ultrafine particle as the precursor of the nickel ultrafine particle. Based on microscopic observation of both the product and the raw material, the influence of the characteristics of the W/O emulsion and experimental conditions on the structure of produced particles was investigated.

\section{Experimental}

\section{W/O emulsion}

As the raw material, $\mathrm{Ni}\left(\mathrm{NO}_{3}\right)_{2} \cdot 6 \mathrm{H}_{2} \mathrm{O}$ (Kanto Chem. Co., Ltd.) was dissolved in water and an aqueous solution of a certain concentration was prepared. Kerosene dissolving sorbitan mono-palmitic acid ester (Sigma-Aldrich Japan Co., Ltd.), which was a surfactant with 6.7 of hydrophilic-lipophilic balance (HLB) value, was added in the water phase. The mass ratio of kerosene to aqueous solution was set to $7 / 3$ and the surfactant was added 2.1 mass percent of aqueous solution and kerosene mixture.

The mixture was sufficiently stirred using ultrasonic homogenizer (Branson Digital Sonifier S-250D; $20 \mathrm{kHz}$, $150 \mathrm{~W}$ ) and the $\mathrm{W} / \mathrm{O}$ emulsion solution was prepared. The distribution of water phase diameter measured by laser diffraction particle size analyzer (Shimadzu SALD-300 V) is shown in Fig. 1. Due to ultrasonic homogenizer, water phase could be dispersed finer than conventional stirring. [17, 18] Moreover, the distribution range of aqueous solution phase is also narrower than that of droplet size using conventional spray methods $[19,20]$. Median diameter of water phase based on this distribution was about $0.18 \mathrm{~m}$.

\section{Experimental procedure}

Schematic diagram of experimental apparatus is shown in Fig. 2. The apparatus consists of an atomizer for W/O emulsion, a thermal decomposition furnace and a particle collection device. The furnace consists of a stainless steel tube with an inner diameter of $40 \mathrm{~mm}$ to which an electric heater with a height of $1200 \mathrm{~mm}$ is attached and a combustion chamber connected to bottom of the tube. Using the pressure atomization nozzle, the emulsion was supplied to the combustion chamber at $9.2 \mathrm{ml} / \mathrm{min}$, which includes about $2.1 \mathrm{ml} / \mathrm{min}$ of the aqueous solution. To set fuel equivalence ratio of 0.75 , which is suitable for stable combustion of $\mathrm{W} / \mathrm{O}$ emulsion, oxygen-enriched air with an oxygen concentration of $26 \mathrm{vol} \%$ was supplied into the combustion chamber at $65 \mathrm{l} / \mathrm{min}$. Moreover, the temperature of the heater was set to $973 \mathrm{~K}$. Fine particles produced in the reactor were collected using an electric precipitator. Temperature of the

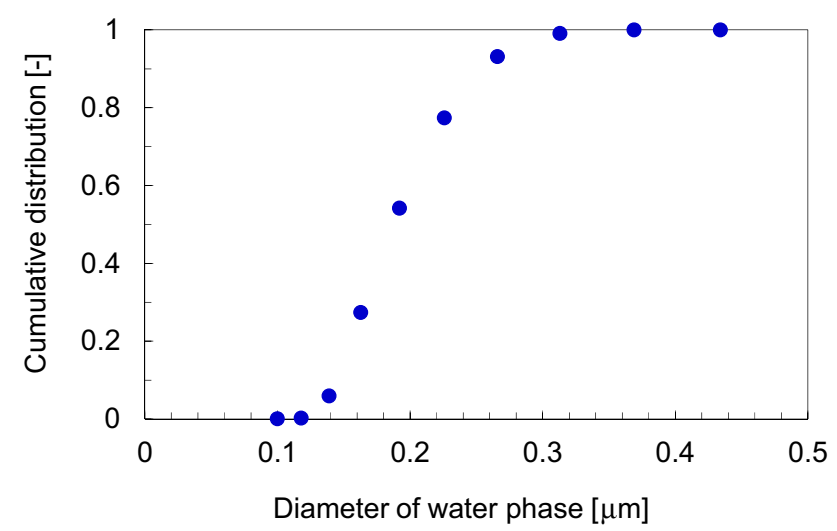

Fig. 1 Distribution of water phase size in W/O emulsion 


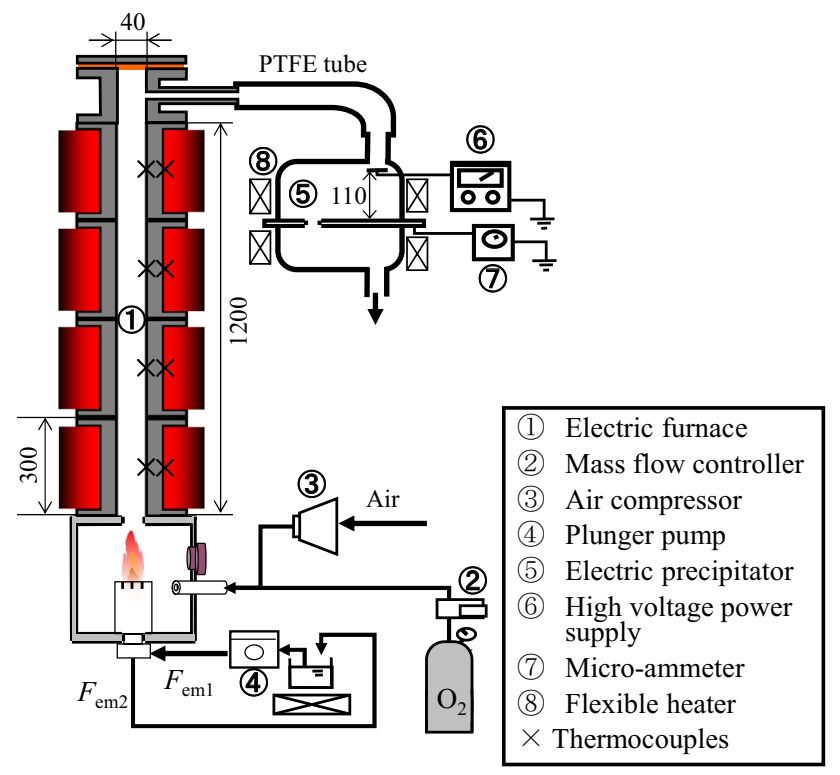

Fig. 2 Schematic diagram of experimental apparatus

electric precipitator was maintained at relatively high temperature conditions where water vapor does not condense. The obtained fine particles were observed by scanning and transmission electron microscopy (SEM: Hitachi Ltd. S 570, TEM: Hitachi Ltd. H 800) and identified using X-ray diffraction pattern (XRD: Shimadzu XRD-6100).

\section{Results and discussion}

\section{Thermogravimetric analysis}

Before fine particle production by W/O emulsion combustion, thermogravimetric analysis (Shimadzu TGA-50) for $\mathrm{Ni}\left(\mathrm{NO}_{3}\right)_{2} \cdot 6 \mathrm{H}_{2} \mathrm{O}$ was carried out to investigate the thermal decomposition properties. These results are shown in Fig. 3. Here, nitrogen, air, oxygen and hydrogen were used as carrier gas, respectively. The flow rates were all $50 \mathrm{ml} / \mathrm{min}$. Moreover, heating rate of all analyses was $1 \mathrm{~K} / \mathrm{min}$.

Weight loss of the hydrated metal salt progressed through almost three stages in any condition. The first two steps would be elimination reaction of hydration water since the mass ratio of the water to the hydrated metal salt (37\%) was roughly consistent with the mass yield at the end of first two steps. Furthermore, the weight loss rate at the end of second step was almost same in any case. The third step would be decomposition of the metal salt and especially reduction reaction under hydrogen atmosphere. In fact, the mass ratios of both nickel oxide and metal nickel to the hydrated metal salt are 26 and $20 \%$, respectively. These values were almost same to the mass yield at the end of third step; it was found

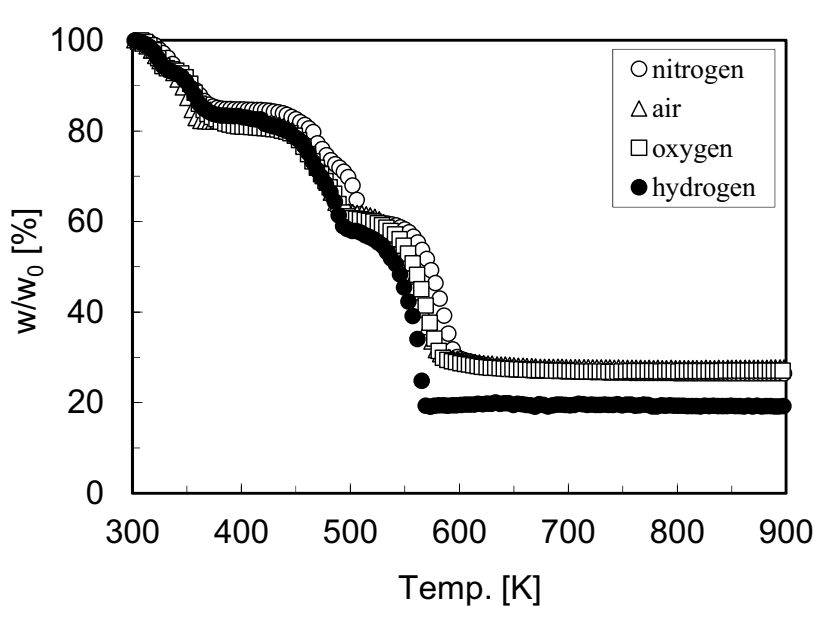

Fig. 3 Thermogravimetric curves for $\mathrm{Ni}\left(\mathrm{NO}_{3}\right)_{2} \cdot 6 \mathrm{H}_{2} \mathrm{O}$

that nickel oxide and/or metal nickel would be produced by decomposition and/or reduction reaction of the metal salt. Moreover, weight loss hardly appeared over $600 \mathrm{~K}$. Based on these results, the above experimental conditions were determined.

\section{Production of nickel oxide particle by $\mathrm{W} / 0$ emulsion combustion}

Due to combustion of W/O emulsion containing precursor solution, particles synthesized in the reactor were obtained. The appearance of these particles was not green, which is the color of nickel oxide (II), but black and carbon deposition during the particle synthesis was supposed. SEM and TEM micrographs of the obtained fine particles are shown in Figs. 4 and 5, respectively. Here, SEM and TEM images of particles made only from aqueous solution are also shown in both figures. According to SEM observation, relatively large spherical particles of more than $5 \mathrm{~m}$ diameter were confirmed in produced materials using low concentration solution. The size of spherical particles increases with increase in the concentration of solution and these large particles appear to contain hollow particles. On the other hand, particles made from high concentration solution $(0.45 \mathrm{M})$ were comparatively small and almost less than $5 \mathrm{~m}$. These large spherical particles were weakly agglomerated and could be dispersed into primary particles in alcohol solvent. In contrast, large spherical particles were hardly observed when only aqueous solution was used. According to TEM observation, to clarify the appearance of primary particles, the diameter of particles produced from W/O emulsion was almost $10 \mathrm{~nm}$ and it appeared that the particles included not only spherical but also cubic shapes. Moreover, the size of particles produced from W/O emulsions was almost same in spite of different concentration of nickel salt solutions. Apart 


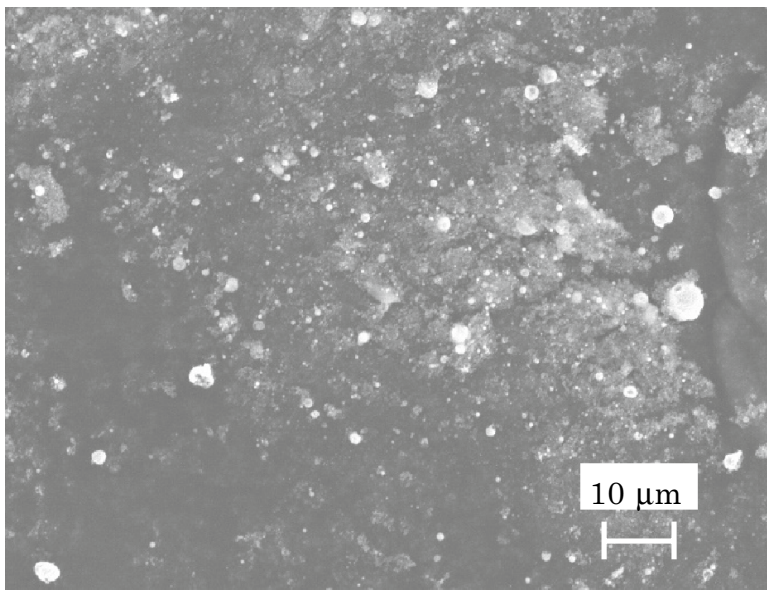

(a) $0.02 \mathrm{M}, \mathrm{W} / \mathrm{O}$ emulsion

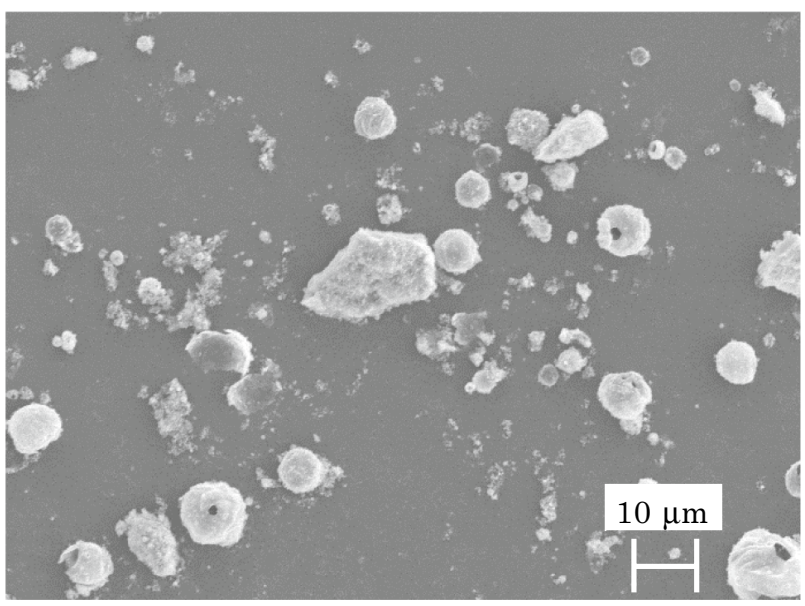

(b) $0.15 \mathrm{M}, \mathrm{W} / \mathrm{O}$ emulsion

Fig. 4 SEM photographs of produced nickel oxide particles

from overlapping of particles, several small black spots were observed in these TEM images. These black spots could indicate crystal of metal nickel according to a diffraction image of the TEM. On the other hand, the particles produced from aqueous solution were larger than those from W/O emulsions and the diameters were approximately $500 \mathrm{~nm}$.

Based on these TEM images, particle size distributions using W/O emulsion as raw materials were determined and shown in Fig. 6. Moreover, median diameter of produced particles based on this distribution is shown in Table 1. Here, all lines were estimated from water phase diameter distributions in W/O emulsions (Fig. 1) and the concentration of the aqueous solution, when it was assumed that one particle was made from one dispersion solution phase. The calculating equation is as follows:

$D_{\mathrm{p}}=\sqrt[3]{\frac{x D_{\mathrm{em}}^{3} M_{\mathrm{p}}}{\rho_{\mathrm{p}}}}$

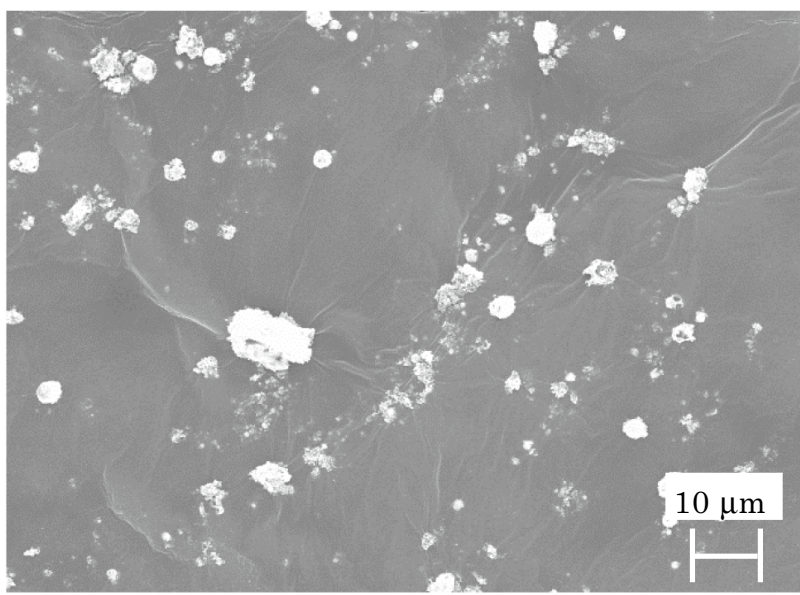

(c) $0.45 \mathrm{M}, \mathrm{W} / \mathrm{O}$ emulsion

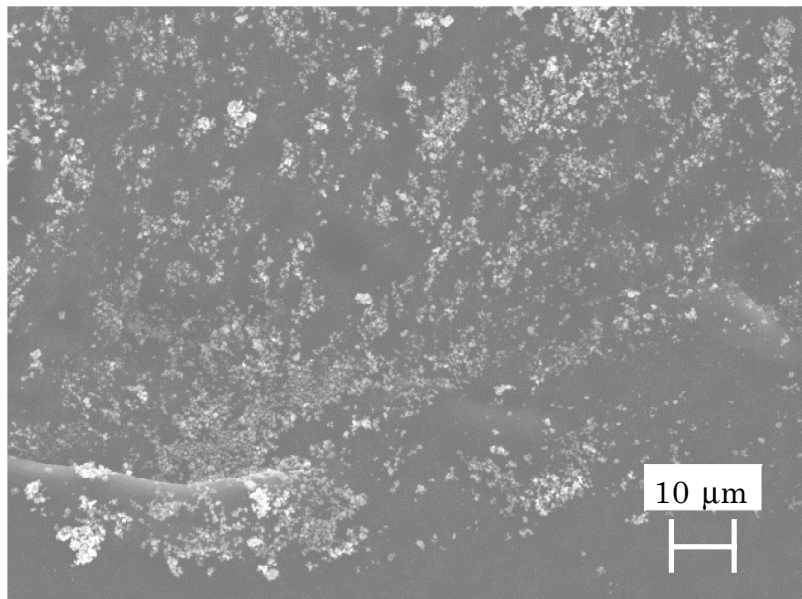

(d) $0.15 \mathrm{M}$, aqueous solution only

From these results, it is observed that the concentration of nickel salt solution had little influence on the size distribution of the synthesized particles although the predicted distributions of particle size shifted to smaller particle size with decrease in the concentration. In comparison with the estimated distributions, it was found that all actual distributions were proximate to the estimate based on the most dilute concentration of aqueous solution. When the concentration is high, solid phase such as metal salt will rapidly precipitate in the solution droplet. It is supposed that the solid phase may play a role in promoting evaporation such as boiling stone. Therefore, the water phase in the emulsion may explosively evaporate and cause secondary atomization both the emulsion and the water phase $[21,22]$ with increase in the concentration. As a result, the higher the concentration of the solution was, the smaller both primary and agglomerated particle would become. Moreover, crystals of nickel oxide (II) have face-centered 


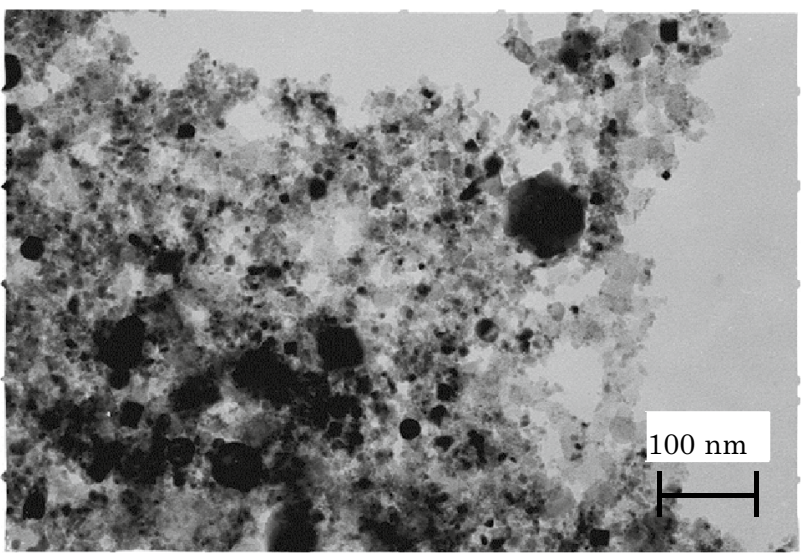

(a) $0.02 \mathrm{M}, \mathrm{W} / \mathrm{O}$ emulsion

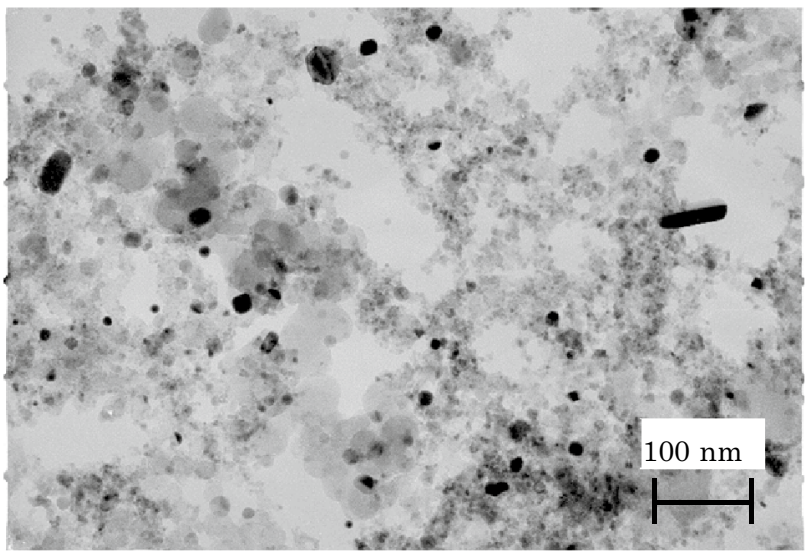

(b) $0.15 \mathrm{M}, \mathrm{W} / \mathrm{O}$ emulsion

Fig. 5 TEM photographs of produced nickel oxide fine particles

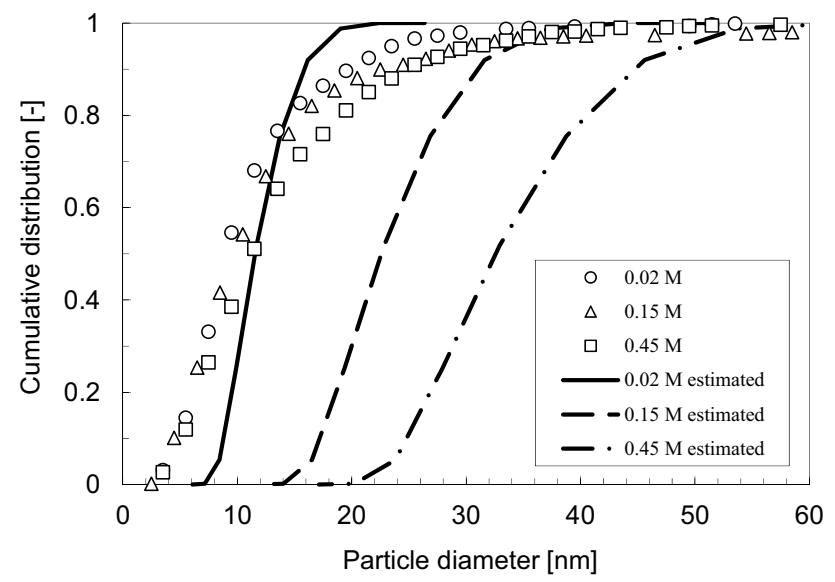

Fig. 6 Particle size distribution of nickel oxide primal particles produced from W/O emulsions

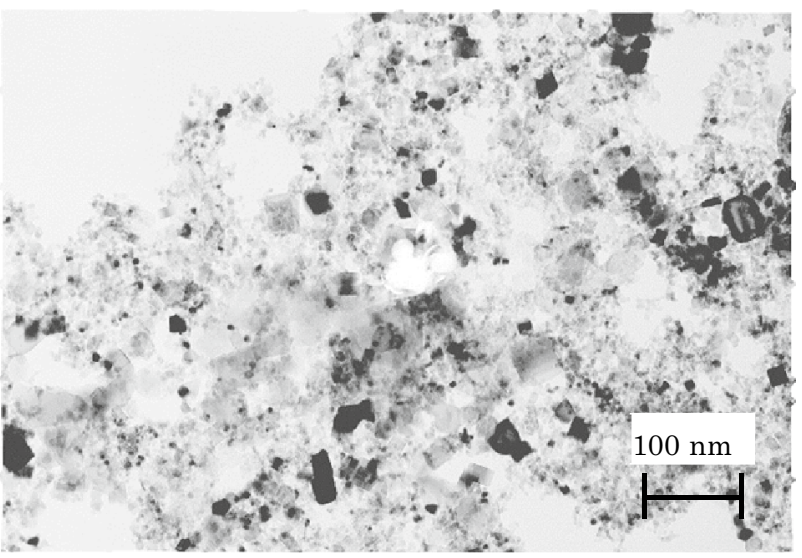

(c) $0.45 \mathrm{M}, \mathrm{W} / \mathrm{O}$ emulsion

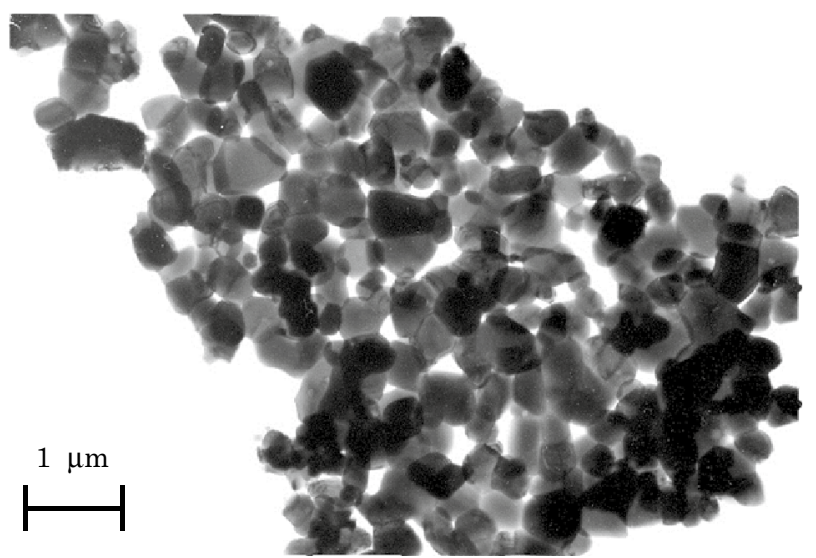

(d) $0.15 \mathrm{M}$, aqueous solution only

Table 1 Median diameter of particles produced from W/O emulsions

\begin{tabular}{llll}
\hline & \multicolumn{2}{l}{ Concentration } & \\
\cline { 2 - 4 } & $0.02 \mathrm{M}$ & $0.15 \mathrm{M}$ & $0.45 \mathrm{M}$ \\
\hline $\begin{array}{c}\text { Median diameter } \\
(\mathrm{nm})\end{array}$ & 12.0 & 12.4 & 14.6 \\
$\begin{array}{c}\text { Estimated median } \\
\text { diameter (nm) }\end{array}$ & 13.0 & 25.4 & 36.7 \\
\hline
\end{tabular}

cubic structure and that is responsible for cubic shape particles observed by TEM.

The results of XRD analysis are shown in Fig. 7. Here, XRD pattern of nickel oxide test reagent is also shown in this figure. XRD pattern of produced particles was not only consistent with that of the test reagent but also showed the other peaks due to existence of metal nickel. From the appearance of the obtained particles, it was suggested that these particles included a mix of carbon substance, but the analysis result of XRD did not show existence of carbon. 


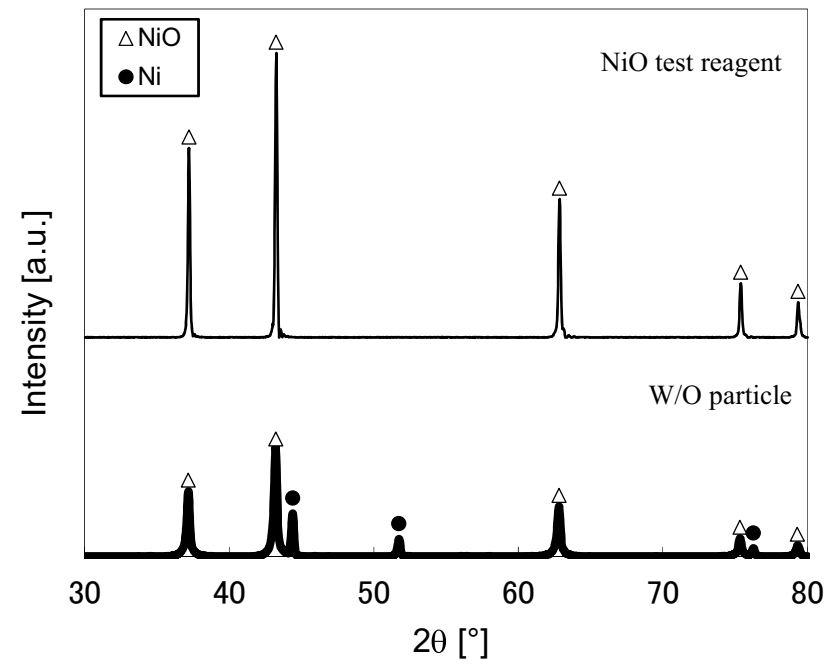

Fig. 7 X-ray diffraction pattern of produced fine particles (solution concentration: $0.45 \mathrm{M}$ )

Therefore, it appears that the carbon substance is probably amorphous material. On the other hand, metal nickel containing in the produced particles was confirmed even under fuel lean combustion conditions. That indicates generation of reducing gases around the produced particles and abovementioned explosive combustion of W/O emulsion could account for instantaneous and local reducing atmosphere. However, residence time in the reactor is very short less than $1 \mathrm{~s}$ and further investigation into detail is required to discuss the reduction reaction of produced particles.

\section{Conclusion}

W/O emulsion combustion for production of ultrafine nickel oxide particles was performed and the characteristics of production method were investigated. As a result, the following was clarified:

1. The diameter of primary particles produced from $\mathrm{W} / \mathrm{O}$ emulsion was approximately $10 \mathrm{~nm}$ based on TEM observation. On the other hand, the size of agglomerated particles was almost less than $10 \mathrm{~m}$ due to SEM observation. The form of the primary particles was spherical or cubic.

2. The size distributions of primary particles were independent of the concentration of nickel salt solutions.

3. Not only nickel oxide but also metal nickel particles were produced by W/O emulsion combustion even under oxidative atmosphere according to TEM observation and XRD analysis.
Acknowledgements This research was partially supported by JSPS KAKENHI Grant Number JP14750616 and Tanikawa Fund Promotion of Thermal Technology. Moreover, we thank Mr. Takanori Watanabe, Mr. Goshi Yokota and Mr. Shingo Kawagoe for assistance with experiments.

Author contribution JK conceived of the presented idea. JK wrote the manuscript with support from NK and YI. MH supervised the project. All authors discussed the results and contributed to the final manuscript.

Open Access This article is distributed under the terms of the Creative Commons Attribution 4.0 International License (http://creativeco mmons.org/licenses/by/4.0/), which permits unrestricted use, distribution, and reproduction in any medium, provided you give appropriate credit to the original author(s) and the source, provide a link to the Creative Commons license, and indicate if changes were made.

\section{References}

1. Medeiros, P.N., Santiago, A.A.G., Ferreira, E.A.C., Li, M.S., Longo, E., Bomio, M.R.D., Motta, F.V.: Influence Ca-doped SrIn2O4 powders on photoluminescence property prepared one step by ultrasonic spray pyrolysis. J. Alloy. Compd. 747, 10781087 (2018)

2. Krasnikova, I.V., Mishakov, I.V., Bauman, Y.I., Karnaukhov, T.M., Vedyagin, A.A.: Preparation of NiO-CuO-MgO fine powders by ultrasonic spray pyrolysis for carbon nanofibers synthesis. Chem. Phys. Lett. 684, 36-38 (2017)

3. Lee, S., Schneider, K., Schumann, J., Mogalicherla, A.K., Pfeifer, P., Dittmeyer, R.: Effect of metal precursor on $\mathrm{Cu} / \mathrm{ZnO} / \mathrm{Al}_{2} \mathrm{O}_{3}$ synthesized by flame spray pyrolysis for direct DME production. Chem. Eng. Sci. 138, 194-202 (2015)

4. Okuyama, K., Lenggoro, I.W.: Preparation of nanoparticles via spray route. Chem. Eng. Sci. 58, 537-547 (2003)

5. Kelder, E.M., Nijs, O.C., Schoonman, J.: Low-temperature synthesis of thin films of YSZ and $\mathrm{BaCeO}_{3}$ using electrostatic spray pyrolysis (ESP). Solid State Ionics 68, 5-7 (1994)

6. Zaouk, D., Zaatar, Y., Asmar, R., Jabbour, J.: Piezoelectric zinc oxide by electrostatic spray pyrolysis. Microelectr. J. 37, 12761279 (2006)

7. Yurteri, C.U., Hartman, R.P.A., Marijnissen, J.C.M.: Producing pharmaceutical particles via electrospraying with an emphasis on nano and nano structured particles-a review. KONA Powder Part. J. 28, 91-115 (2010)

8. Okuyama, K., Lenggoro, I.W., Tagami, N.: Preparation of $\mathrm{ZnS}$ and $\mathrm{CdS}$ fine particles with different particle sizes by a spray-pyrolysis method. J. Mater. Sci. 32, 1229-1237 (1997)

9. Kang, Y.C., Lenggoro, I.W., Park, S.B., Okuyama, K.: YAG: Ce phosphor particles prepared by ultrasonic spray pyrolysis. Mater. Res. Bull. 35, 789-798 (2000)

10. Lenggoro, I.W., Itoh, Y., Iida, N., Okuyama, K.: Control of size and morphology in $\mathrm{NiO}$ particles prepared by a low-pressure spray pyrolysis. Mater. Res. Bull. 38, 1819-1827 (2003)

11. Wang, W.N., Lenggoro, I.W., Terashi, Y., Kim, T.O., Okuyama, K.: One-step synthesis of titanium oxide nanoparticles by spray pyrolysis of organic precursors. Mater. Sci. Eng., B 123, 194-202 (2005)

12. Watanabe, T., Nawata, M., Kobayashi, J., Kobayashi, N., Hasatani, M.: Synthesis of Y2O3: Eu nanoparticles by emulsion combustion at high temperature. Kagaku Kogaku Ronbunshu 34, 181-186 (2008). (in Japanese) 
13. Takatori, K., Tani, T., Watanabe, N., Kamiya, N.: Preparation and characterization of nano-structured ceramic powders synthesized by emulsion combustion method. J. Nanopart. Res. 1, 197-204 (1999)

14. Tani, T., Watanabe, N., Takatori, K.: Emulsion combustion and flame spray synthesis of zinc oxide/silica particles. J. Nanopart. Res. 5, 39-46 (2003)

15. Tani, T., Watanabe, N., Takatori, K.: Morphology of oxide particles made by the emulsion combustion method. J. Am. Ceram. Soc. 86, 898-904 (2003)

16. Tolosa, L.I., Forgiarini, A., Moreno, P., Salager, J.L.: Combined effects of formulation and stirring on emulsion drop size in the vicinity of three-phase behavior of surfactant-oil water systems. Ind. Eng. Chem. Res. 45, 3810-3814 (2006)

17. Rao, J., McClements, D.J.: Formation of flavor oil microemulsions, nanoemulsions and emulsions: influence of composition and preparation method. J. Agric. Food Chem. 59, 5026-5035 (2011)

18. Lin, C.Y., Chen, L.W.: Comparison of fuel properties and emission characteristics of two- and three-phase emulsions prepared by ultrasonically vibrating and mechanically homogenizing emulsification methods. Fuel 87, 2154-2161 (2008)
19. Wang, W.N., Purwanto, A., Lenggoro, I.W., Okuyama, K., Chang, H.K., Jang, H.D.: Investigation on the correlations between droplet and particle size distribution in ultrasonic spray pyrolysis. Ind. Eng. Chem. Res. 47, 1650-1659 (2008)

20. Jaworek, A., Sobczyk, A.T.: Electrospraying route to nanotechnology: an overview. J. Electrostat. 66, 197-219 (2008)

21. Lin, C.Y., Chen, L.W.: Engine performance and emission characteristics of three-phase diesel emulsions prepared by an ultrasonic emulsification method. Fuel 85, 593-600 (2006)

22. Li, Y., Wang, T., Liang, W., Wu, C., Ma, L., Zhang, Q., Zhang, X., Jiang, T.: Ultrasonic preparation of emulsions derived from aqueous bio-oil fraction and 0\# diesel and combustion characteristics in diesel generator. Energ. Fuel. 24, 1987-1995 (2010)

Publisher's Note Springer Nature remains neutral with regard to jurisdictional claims in published maps and institutional affiliations. 